\title{
Electric field dependent EL2 capture coefficient in semi-insulating GaAs obtained from propagating high field domains
}

\author{
F. Piazza, P. C. M. Christianen, and J. C. Maan ${ }^{\text {a) }}$ \\ High Field Magnet Laboratory and Research Institute for Materials, University of Nijmegen, Toernooiveld, \\ 6525 ED Nijmegen, The Netherlands
}

(Received 19 December 1995; accepted for publication 16 July 1996)

\begin{abstract}
We have determined the electric field dependence of the carrier capture coefficient of the EL2 traps in semi-insulating GaAs up to $7 \mathrm{kV} / \mathrm{cm}$ by means of the quantitative analysis of propagating high electric field domains. The experimental data show a trapping coefficient which is proportional to the electron drift velocity, supporting the microscopic model of electric field enhanced trapping of carriers over a configurational barrier. (c) 1996 American Institute of Physics.
\end{abstract}

[S0003-6951(96)03639-X]

Liquid encapsulated Czochralski (LEC) grown GaAs, a material commonly used as a substrate in device fabrication (metal-semiconductor field-effect transistor, gamma ray spectrometers), is semi-insulating (SI) by a compensation of residual shallow donor and acceptor charge by trapped charge on native EL2 deep traps, usually attributed to an $\mathrm{As}_{\mathrm{Ga}}$ antisite defect. ${ }^{1}$ At room temperature the Fermi level is pinned at the EL2 midgap energy level, which is partially ionized and in equilibrium with electrons in the conduction band via trapping (coefficient $c_{n}$ ) and emission (rate $e_{n}$ ). If the system is driven out of equilibrium by an external driving force, for instance a large electric field, the electron distribution is no longer described by the Boltzmann statistic ${ }^{5}$ and the trapping coefficient and emission rate are modified. In SI GaAs the trapping coefficient is found to increase with electric field, leading to negative differential resistance, ${ }^{2}$ which is responsible for noise in devices based on SI GaAs substrates, ${ }^{3-5}$ low frequency current oscillations and high field domain formation. ${ }^{6-8}$ Theoretically the field enhanced trapping is explained both by a configurational barrier of 60 $\mathrm{meV}$ due to a multiphonon capture process which requires an electric field of about $0.5 \mathrm{kV} / \mathrm{cm}$ to be overcome ${ }^{9}$ or by an enhanced capture of hot electrons in the $L$ valley which occurs at about $3.0 \mathrm{kV} / \mathrm{cm} .{ }^{10}$ Experimentally, the field dependence of $c_{n}$ is poorly known. Prinz et al. ${ }^{11}$ have measured an enhancement of more than one order of magnitude of the capture cross section $\sigma_{n}$ for $E=10 \mathrm{kV} / \mathrm{cm}$ as compared to the zero field value, which implies the enhancement of the trapping coefficient determined by $c_{n}=\sigma_{n}\left\langle v_{t h}\right\rangle$, where $\left\langle v_{t h}\right\rangle$ is the electron thermal velocity, which almost doubles in high fields. However, the $c_{n}(E)$ dependence of the full range of electric fields is not available. The reason for this lack of data is that, for short samples (less than one millimeter), incontrollable contact effects are important, while for long samples propagating high electric field domains are formed.

We will show in this letter that, if the local electric field and charge density of such propagating domains are measured locally and quantitatively, $c_{n}$ as a function of the electric field can be calculated even if the sample is electrically instable.

${ }^{a)}$ Electronic mail: maan@sci.kun.nl
Experimental data are obtained with a set-up based upon the longitudinal electro-optic effect of a $\mathrm{Bi}_{12} \mathrm{SiO}_{20}$ (BSO) crystal which transforms a voltage distribution in a light intensity distribution. ${ }^{12}$ The crystal, with a transparent electrode on the front and a dielectric mirror on the back side, is placed on top of the sample. The light of an expanded polarized pulsed laser beam enters the crystal through the transparent electrode, is reflected back by the dielectric mirror and analyzed. Grounding the top electrode of the BSO, the phase shift induced by the voltage difference between the two sides of the crystal corresponds to the voltage distribution on the sample and can be recorded as a light intensity distribution on a charge-coupled device camera (maximum speed of 1 $\mathrm{kHz}$ ). The voltage is directly obtained from the light intensity via an in situ calibration. A $\langle 100\rangle$ grown LEC single crystal GaAs wafer, nominally undoped, with a thickness of $0.5 \mathrm{~mm}$ and with two bar NiAuGe contacts $8 \mathrm{~mm}$ apart is used as a sample. The shallow acceptor density (mainly carbon and zinc), the shallow donor density (mainly silicon), the free electron density at room temperature at zero electric field and the mobility are respectively $N_{A}=2.2 \times 10^{15} \mathrm{~cm}^{-3}, \quad N_{D}=1.3 \times 10^{15} \mathrm{~cm}^{-3}, \quad n_{0}=1.9$ $\times 10^{7} \mathrm{~cm}^{-3}$ and $\mu_{n}=6600 \mathrm{~cm}^{2} / \mathrm{Vs}$. The density of EL2 states is estimated to be $N_{\mathrm{EL} 2}=1.3 \times 10^{16} \mathrm{~cm}^{-3}{ }^{1}$ The occu-

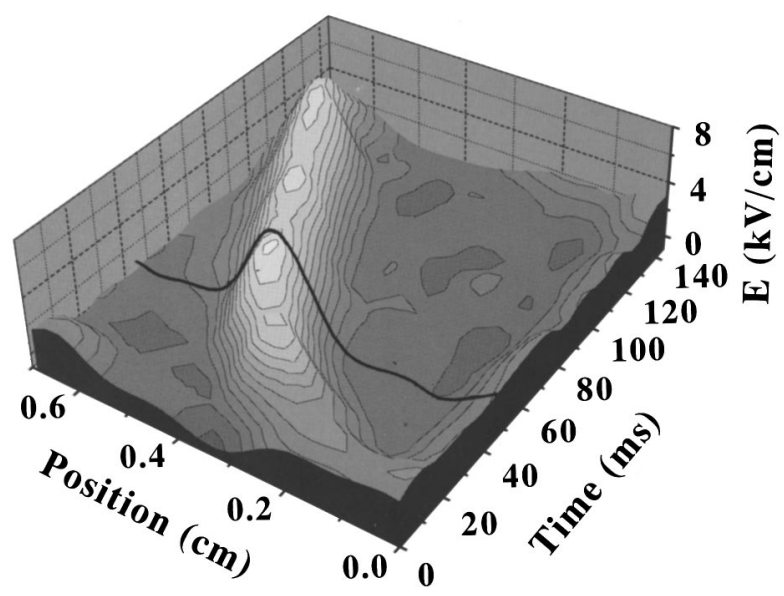

FIG. 1. Electric field as function of time and of the distance from the cathode describing the spontaneous propagation of a high field domain with an applied voltage of $1.6 \mathrm{kV}$. The thick line marks the electric field distribution as a function of the position that is reported in Fig. 2. 


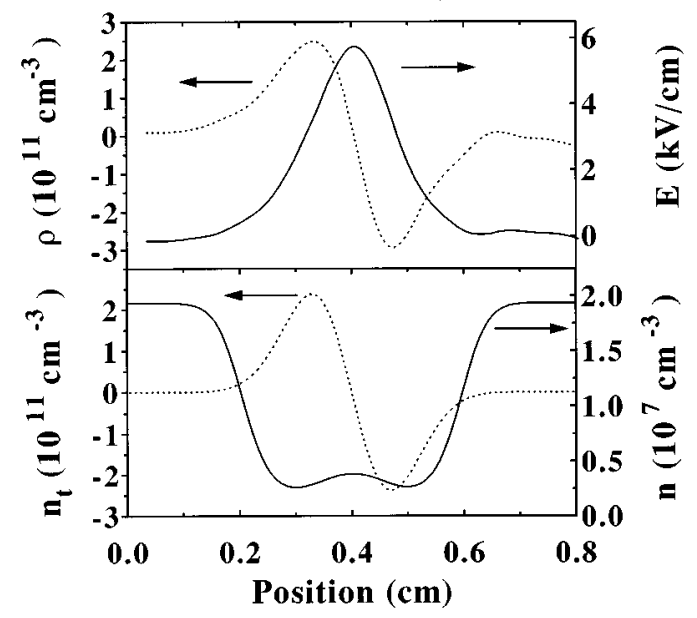

FIG. 2. Top: measured electric field and charge distribution as a function of the position for an applied voltage of $1.4 \mathrm{kV}$. Bottom: evaluation of the conduction band electron density $n$ and of the density fluctuation of the EL2 trapped electron $n_{t}$ from the data on top.

pation densities at thermodynamic equilibrium fix only the ratio between $c_{n}$ and $e_{n}$; using the value of $\sigma_{n}$ at zero electric field ${ }^{11,13}$ with $\left\langle v_{t h}\right\rangle=2 \times 10^{7} \mathrm{~cm} / \mathrm{s}$, we find $c_{n}=2.0 \times 10^{-8} \mathrm{~cm}^{3} / \mathrm{s}$ and $e_{n}=5.4 \times 10^{-2} \mathrm{~s}^{-1}$, which is consistent with literature values.

For dc bias voltages over the contacts below $0.6 \mathrm{kV}$, the sample behaves ohmic, while for higher voltages the current oscillates periodically between two levels $(0.1$ and $10 \mathrm{~Hz})$, showing that propagating high field domains are formed. In Fig. 1 the electric field profile of a domain obtained by differentiation of the measured voltage distribution is shown as a function of time and position for an applied voltage of 1.6 $\mathrm{kV} / \mathrm{cm}$. The domain forms at the cathode, initially grows and slows down while propagating in the bulk and finally reaches a stable configuration which advances towards the anode with the same shape and constant velocity $v_{d}$. When the domain arrives at the anode, it is annihilated, a new domain is formed at the cathode, and the process starts again. Stable domains with the same width but with a linearly increasing electric field peak and domain velocity are observed in the range of $1.3-1.9 \mathrm{kV}$. For voltages larger than $1.9 \mathrm{kV}$ the domain is annihilated at the anode before reaching its stable shape and chaotic behavior sets in.

Stable propagating domains are the ideal tool to obtain information about the electric field dependence of the trapping coefficient because (i) a wide range of electric fields is simultaneously present in the sample $(0.4-7.5 \mathrm{kV} / \mathrm{cm}$ ), (ii) contact effects are unimportant and (iii) the constant velocity and shape allows us to combine the time and space dependence of the basic equations underlying the behavior of the slow domains in a single variable by the transformation $f(x, t) \rightarrow f\left(x-v_{d} t\right)$. Using Poisson's equation, we observe that the space charge density distribution $\rho(x)$ (Fig. 2) is a dipole with a maximum value of $2 \times 10^{11} \mathrm{~cm}^{-3}$. This value is much higher than the free electron density in the conduction band and can only be explained by considering the generation-recombination dynamics of the electrons in the EL2 levels, which are thus essential for the domain formation. The behavior of the free electrons in the domain can be

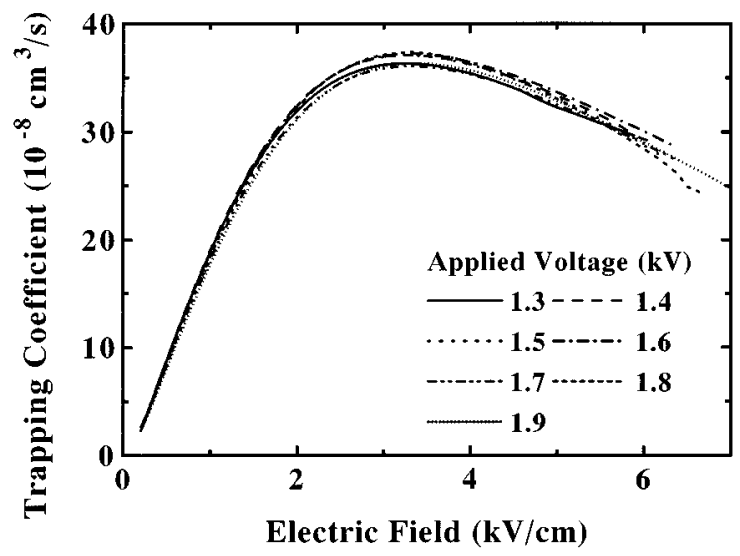

FIG. 3. Electron trapping coefficient in EL2 levels as a function of the electric field obtained for domains at seven different applied voltages.

evaluated from the current continuity equation,

$$
J_{e x t}-v_{d} \rho(x)=\left(n(x) v_{n}(E)+D_{n} \frac{\partial n(x)}{\partial x}\right),
$$

where the external current $J_{\text {ext }}$ and $\rho(x)$ are experimentally determined, the field dependent electron drift velocity $v_{n}$ and the diffusion coefficient $D_{n}$ are obtained from literature ${ }^{14}$ and $n(x)$ is the only unknown quantity. Once $n(x)$ is known, the fluctuation $n_{t}(x)$ around the equilibrium value of the density of electrons trapped in EL2 levels is easily obtained as $\rho(x)=n_{0}-n(x)+n_{t}(x)$. In Fig. 2 we present the measured electric field and charge distribution with an applied voltage of $1.6 \mathrm{kV}$ and the corresponding $n(x)$ and $n_{t}(x)$ profiles. In determining $n(x)$ we used Eq. 1 and $v_{n}(E)$ as given for ultrapure GaAs, which is a good approximation for our sample because at room temperature the mobility is limited by electron-phonon interaction. ${ }^{15}$

The high resistivity of the domain is found to be caused by a $80 \%$ depletion of the conduction band electrons, while the charge dipole which is necessary to sustain the domain is almost entirely due to fluctuations in the occupation of EL2 levels. Although $n_{t}$ is four orders of magnitude larger than

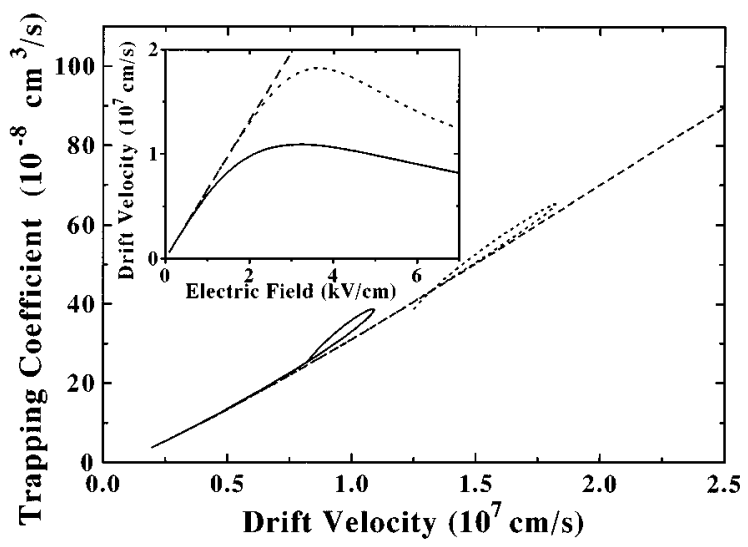

FIG. 4. Trapping coefficient as function of drift velocity evaluated for the three different electric field dependencies of the drift velocity that are plotted in the insert. The continuous line is taken from Ref. 12, the dotted line from Ref. 15 and the dashed line is obtained with a constant mobility. The presence of loops is a consequence of the negative slope of the drift velocity for the different models. 
$n$, it is still five orders of magnitude smaller than the density of occupied states in equilibrium. Therefore the variation in the ratio between electrons in conduction band and electrons in EL2 levels is practically completely determined by $n$ only and the reduction of $n$ in the region of high field is a direct evidence for the enhanced trapping. It is important to note that in Eq. 1 all the possible sources of nonlinearity in the $J-E$ curve are taken into consideration and the experiment directly shows the contribution by the electric field enhanced trapping through the decrease of $n$ at high electric fields. In particular, the contribution from the diffusion is negligible and the low value of $v_{d}$ makes the displacement current not relevant for domain formation, contrary to Gunn domains, where this is the major effect. ${ }^{2}$

We can obtain the electric field dependence of $c_{n}$ from the rate equation for $n_{t}$ :

$$
v_{d} \frac{\partial n_{t}}{\partial t}=c_{n}\left(k N_{\mathrm{EL} 2}+n_{t}\right) n-e_{n}\left[(1-k) N_{\mathrm{EL} 2}-n_{t}\right],
$$

where $k=\left(N_{\mathrm{EL} 2}-N_{A}+N_{D}\right) / N_{\mathrm{EL} 2}$ is the compensation ratio, considering $e_{n}$ constant up to electric fields in the order of $10^{5} \mathrm{~V} / \mathrm{cm}$, when the Poole-Frankel effect becomes important. ${ }^{16} c_{n}$ has been evaluated for seven applied voltages (Fig. 3) and it is very satisfactory that, while the peak electric field varies by a factor of two and $v_{d}$ by a factor of four, a unique curve is obtained, which proves the consistency of our analysis.

The trapping coefficient is seen to increase almost linearly by a factor of fifteen for electric fields on the order of 3 $\mathrm{kV} / \mathrm{cm}$, where it reaches a maximum after which it slowly decreases. The actual $c_{n}$ curve depends strongly on the behavior of $v_{n}$ in Eq. 1 and in particular the maximum of $c_{n}$ is a direct consequence of the bending of $v_{n}$ due to the scattering of electrons between $\Gamma$ and $L$ minima. To obtain a relation that is less direct but that relies only on experimental evidence, we plot $c_{n}$ as function of $v_{n}$ evaluated for different $v_{n}(E)$ dependencies ${ }^{14,17}$ in Eq. 1 (Fig. 4).

The plot shows (i) that the trapping coefficient is linearly dependent on the carrier drift velocity, (ii) that the enhance- ment of the trapping starts already at a drift velocity much lower than that needed for intervalley scattering, showing that the electrons have to overcome a much smaller barrier, and (iii) that these results are not determined by the way the drift velocity is assumed to depend on the electric field, for any reasonable value. The configurational barrier due to multi-phonon trapping, which requires an electric field of only $0.5 \mathrm{kV} / \mathrm{cm}$ for the onset of electron capture, is fully consistent with our experimental results.

In summary, we have presented a time and space resolved measurement of propagating high field domains in SI-GaAs which allows a direct evaluation of the electric field dependence of $c_{n}$. We show that $c_{n}$ depends linearly on the carrier drift velocity and that, as function of the electric field, it shows an increase at an electric field that is much lower than necessary for intervalley scattering, and this fact strongly supports the configurational barrier model.

${ }^{1}$ D. C. Look, Semicond. Semimet. 38, 91 (1993).

${ }^{2}$ E. Schöll, Nonequilibrium Phase Transitions in Semiconductors (Springer, Berlin, 1987).

${ }^{3}$ S. P. McAlister, Z.-M. Li, and D. J. Day, Can. J. Phys. 69, 207 (1991).

${ }^{4}$ M. A. Abdela and B. K. Jones, Solid-State Electron. 36, 237 (1993).

${ }^{5}$ D. S. McGregor, R. A. Rokeski, G. F. Knoll, F. L. Terry, J. East, and Y. Eisen, J. Appl. Phys. 75, 7910 (1994).

${ }^{6}$ A. Barraud, C. R. Acad. Sci. 256, 3632 (1963); D. C. Northrop, P. R. Thornton, and K. E. Trezise, Solid-State Electron. 7, 17 (1964).

${ }^{7}$ H. K. Sacks and A. G. Milnes, Int. J. Electron. 28, 565 (1970).

${ }^{8}$ D. A. Johnson, R. A. Puechner, and G. N. Maracas, J. Appl. Phys. 67, 300 (1990).

${ }^{9}$ M. Kaminska, J. M. Parsey, J. Lagowski, and H. C. Gatos, Appl. Phys. Lett. 41, 989 (1982)

${ }^{10}$ Y. V. Vorobev, E. I. Tolpygo, and M. K. Sheinkman, Phys. Status Solidi B 123, 295 (1984).

${ }^{11}$ V. Ya. Prinz and B. A. Boblyev, Sov. Phys. Semicond. 14, 1097 (1981); V. Ya. Prinz and S. N. Rechkunov, Phys. Status Solidi A 118, 159 (1983).

${ }^{12}$ B. Willing and J. C. Maan, Phys. Rev. B 49, 13995 (1994).

${ }^{13}$ J. S. Blakemore, J. Phys. Chem. Solids 49, 627 (1988).

${ }^{14}$ J. C. Ruch and G. S. Kino, Appl. Phys. Lett. 2, 40 (1967).

${ }^{15}$ S. M. Sze, in Physics of Semiconductor Devices (Wiley, Singapore, 1981).

${ }^{16}$ S. Makram-Ebeid and M. Lannoo, Phys. Rev. B 25, 6406 (1982).

${ }^{17}$ C. M. Hurd and W. R. McKinnon, J. Appl. Phys. 75, 598 (1993). 